\title{
Production of functional graphene by kitchen mixer: mechanism and metric development for in situ measurement of sheet size
}

\author{
Zulhelmi Ismail $^{1}$ Abu Hannifa Abdullah ${ }^{2}$ Anis Sakinah Zainal Abidin ${ }^{2}$. \\ Kamal Yusoh ${ }^{2}$
}

Received: 23 May 2017/ Accepted: 8 July 2017/Published online: 19 July 2017

(c) The Author(s) 2017. This article is an open access publication

\begin{abstract}
It has been reported that the production of defect free graphene is possible by the application of a kitchen mixer. Yet, we note that the natural-surfactant role in the exfoliation mechanism by a kitchen mixer has rarely been discussed. To investigate the possibility of graphene exfoliation in a bio-surfactant medium, we have produced graphene from the co-mixing of graphite and gum Arabic. Through the modelling of bulky graphite as a single composite disc, we have shown that the exfoliation of graphite crystal may be possible through rotational motion of graphite surface. In this paper, we also have developed two simple metric systems that were designed from the application of UV spectroscopy for in situ measurement of graphene sheet size after exfoliation step.
\end{abstract}

Keywords Functional graphene - Natural surfactant . Kitchen mixer · Mechanism - In situ measurement

Zulhelmi Ismail

zulhelmiismail.ump@gmail.com

Abu Hannifa Abdullah

abuhaniffa@yahoo.com

Anis Sakinah Zainal Abidin

anissakinah14010@gmail.com

Kamal Yusoh

kamal@ump.edu.my

1 Faculty of Manufacturing Engineering, Universiti Malaysia Pahang, 26600 Pekan, Pahang, Malaysia

2 Faculty of Chemical Engineering and Natural Resources, Universiti Malaysia Pahang, 26300 Kuantan, Pahang, Malaysia

\section{Introduction}

Ever since graphene first isolation from pyrolytic graphite in 2004 [1], the interest of research society on this material is continuously growing. The advancing development of graphene research field is majorly assisted by the interest of society towards unique properties possesses by graphene. Due to the strong mechanistic properties of graphene, it has high potential for application as filler in polymer composite $[2,3]$. High transparency of graphene meanwhile is suitable for manufacturing of transparent conductor [4]. In the membrane technology, super permeability of graphene to water is useful as material for desalination of sea water [5]. Excellent electrical conductivity of graphene meanwhile links graphene to advanced electronic applications such as conductive ink [6] and 2D wire [7].

For introduction of graphene towards industrial application, selection of synthesis route is extremely important. Bottom-up approach such as chemical vapour disposition [8] or epitaxial growth [9] is useful for preparation of high quality grade of graphene. However, the resulting yield from both methods is low and not economical for large scaled production of graphene. To reduce the preparation cost of graphene, a kitchen mixer can be used as exfoliating tool for graphite to graphene [10-14]. This method principally belongs to liquid-phase exfoliation class, which also includes vortex fluidic exfoliation $[15,16]$ and high-pressure driven exfoliation [17-19]. Application of kitchen mixer allows a facile preparation of graphene and the quality of produced graphene is even comparable with the quality of graphene prepared from the lab homogeniser [10]. Moreover, the application of toxic solvent [12] as exfoliating medium in this method can be easily replaced with water-based surfactant [11] or edible protein [13] for bio-friendly and sustainability process. 
In this work, we are interested to investigate the theoretical explanation behind possible exfoliation of graphene in gum Arabic solution by the mixing action of a kitchen mixer [20]. In addition, we want to know the exact component in gum Arabic that may be responsible for stabilisation of graphene against reaggregation in water. Finally, we used UV spectroscopy and TEM to design a metric that may be suitable for rapid characterisation of functional graphene sheet size after exfoliation step. We do believe that our study will assist in the understanding of graphene exfoliation process for mass synthesis of graphene in the future work.

\section{Experimental}

\section{Materials}

Industrial graphite flakes and gum Arabic were purchased from Sigma-Aldrich (Malaysia) and were used as received. Kitchen mixer of Philips brand with nominal power of $800 \mathrm{~W}$ was obtained from a local retail.

\section{Preparation and characterisations of graphene}

We performed the exfoliation of graphene by a co-mixing of graphite $(1 \mathrm{mg} / \mathrm{ml})$ and gum Arabic $(1 \mathrm{mg} / \mathrm{ml})$ in a mixing volume of $400 \mathrm{ml}$. After centrifugation (Heraeus Pico Micro) at $1500 \mathrm{rpm}$ (RCF: 330) for $45 \mathrm{~min}$, we washed the resulting supernatant through multiple filtration steps with ultrapure water (Millipore) before the freezedrying stage. For observation of sheet size change after the increase of exfoliation time, we extracted $1 \mathrm{ml}$ of aliquots from the original volume after every paused interval $(1,3$, 5 and $7 \mathrm{~h}$ ). Absorption coefficient of resulting graphene meanwhile was computed from the redispersion of dried graphene at varied mass $(0.03,0.06,0.09,0.12,0.15 \mathrm{mg} /$ $\mathrm{ml}$ ) and was followed by the absorbance measurement of graphene solution at $660 \mathrm{~nm}$ for each mass by UV spectroscopy (Shidmazu). TEM imaging and size measurement of graphene sheets were conducted on TEM Libra (Zeiss), while Raman measurement was performed on graphite and graphene using Witec Alpha 300R (532 nm). The functionalization study of prepared graphene was possible through the applications of XPS (Ultra Kratos) and IR (Perkin Elmer) on the dried graphene. The stability of exfoliated graphene in water was studied through redispersion of $3 \mathrm{mg}$ of graphene mass into $10 \mathrm{ml}$ of ultrapure water by bath sonication (Branson). Absorbance monitoring of graphene suspension was conducted in every $24 \mathrm{~h}$ for 6 days using UV spectroscopy. For comparison purpose, stability of graphene supernatant after washing stage was also observed from absorbance value within similar time duration. Contact angle measurement meanwhile was determined from the change of droplet size on suspended graphite and graphene using goniometer.

\section{Results and discussion}

\section{Initial characterisation of graphene}

\section{Validation of graphene}

We notice that the presence of highest UV peak for the spectra of washed and unwashed gum Arabic-graphene $\left(G_{\mathrm{GA}}\right)$ in Fig. 1a is at $269 \mathrm{~nm}$. Interestingly, this is a strong indicator for graphene presence and as evidence for successful exfoliation of multilayer by a kitchen mixer [21]. To rule out the effect of gum Arabic presence on the absorbance of graphene, we note that the highest UV peak for gum Arabic is below $200 \mathrm{~nm}$ and definitely is not in the range of wavelength for graphene. We also performed a calculation from the Lambert-Beers law $(A=\alpha \mathrm{Cl})$ for determination of the absorption coefficient $(\alpha)$ value for $G_{\mathrm{GA}}$ at $660 \mathrm{~nm}$ of spectroscopy wavelength.

As shown in Fig. 1b, the obtained value for $\alpha$ is $1210 \mathrm{mg}^{-1} \mathrm{~m}^{-1} \mathrm{ml}$ and this value is definitely in the range with the previous reported $\alpha$ for surfactant-based graphene [22]. However, we note that the determined value of $\alpha$ in this research work is very far from the proposed theoretical $\alpha$ value of $4237 \mathrm{mg}^{-1} \mathrm{~m}^{-1} \mathrm{ml}$ for pristine monolayer graphene [23]. As suggested by theory, the deviation of $\alpha$ may possibly caused by the preparation method, the presence of functional group on graphene sheet and mean thickness of multilayer graphene [24].

\section{Morphological study of graphene}

For visual conformation of graphene presence in the resulting black opaque supernatant, we used transmission electron microscopy (TEM) for imaging of graphene in the drop casted sample. As shown by the example TEM image of graphene in Fig. $2 \mathrm{a}\left(t_{\text {mix }}: 7 \mathrm{~h}\right)$, the appearance of semitransparent sheets is proving the possibility of graphene preparation by a kitchen mixer and gum Arabic/water solution. Moreover, the absence of wrinkles graphene sheets is also suggesting that this method does not introduce any defect on the basal structural of graphene despite long processing duration of mixing $[10,11,21]$. The mean length, $<\mathrm{L}>$ for resulting graphene from $1,3,5$ and $7 \mathrm{~h}$ meanwhile, was measured from TEM by the edge-edge evaluation of 100 visible graphene sheets and included in Fig. 8. For comparison purpose, we have also studied the length distribution of graphene by atomic force microscopy (AFM). It was found that the size of small graphene in 

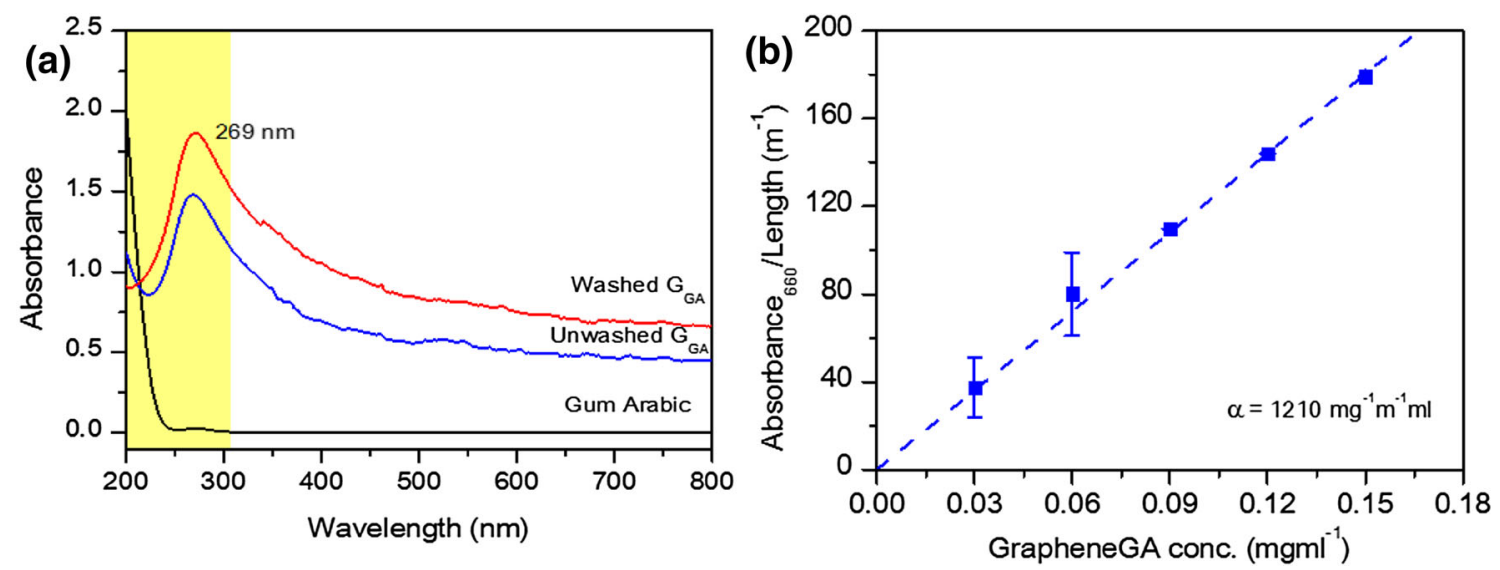

Fig. 1 a Spectrum of gum Arabic, unwashed graphene and washed graphene. b The absorption coefficient value, $\alpha$, for graphene is extracted from the slope of plotted linear

Fig. 2 a High TEM

magnification and $\mathbf{b}$ Low TEM magnification for exfoliated graphene after $7 \mathrm{~h}$ of mixing c AFM analysis on the similar sample was used to study statistical length and thickness distribution of graphene (a)

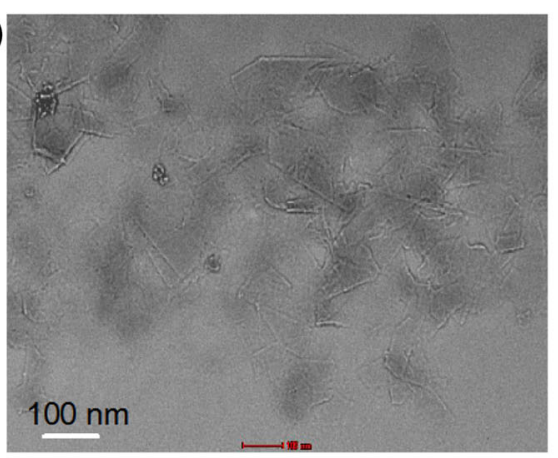

(b)
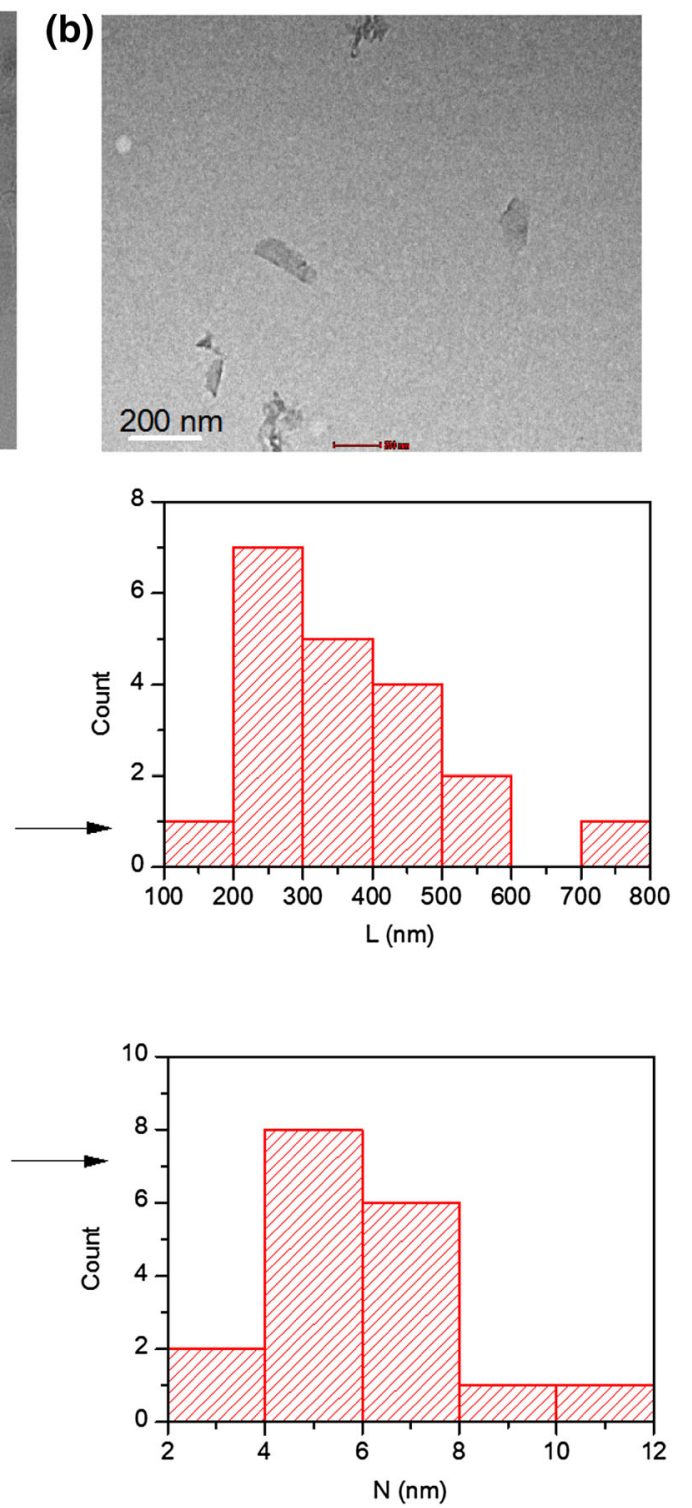
200-300 nm domain was more frequently measured by AFM than TEM in our work. As suggested by the previous report [11], the lack of small graphene count by TEM is normally caused by wide polydispersity of the sample as well as the high transparency of graphene at lower layers number. As only visible graphene sheets were considered for objective measurement of length by TEM, small size graphene that usually is almost invisible in the micrograph was not included in the counting process. As for the mean thickness, $<\mathrm{N}>$ of the produced graphene, the thickness of graphene was evaluated from 20 sheets with thickness value from five layers to twenty-nine layers of sheets (see Fig. 2b).

\section{Defect evaluation of graphene}

We conducted Raman spectroscopy measurement on the $G_{\mathrm{GA}}$ film for investigation of defect after mixing. A rectangle shape film with dimension of $2 \mathrm{~cm} \times 2 \mathrm{~cm}$ was cut from the vacuum-filtered film. As shown from the example of Raman spectrum for graphene in Fig. 3, the presence of intense $\mathrm{G}$ band $\left(1578 \mathrm{~cm}^{-1}\right)$, defectassociated $\mathrm{D}$ band $\left(1347 \mathrm{~cm}^{-1}\right)$ and $2 \mathrm{D}$ band $\left(2684 \mathrm{~cm}^{-1}\right)$ on the Raman spectrum of $G_{\mathrm{GA}}$ can be used to verify the presence of graphene on the vacuumfiltered membrane [10]. A significant Raman shift of G, $\mathrm{D}$ and 2D bands for the graphene from 1576, 1344 and $2701 \mathrm{~cm}^{-1}$ demonstrates the change of graphene structure after exfoliation from bulk graphite. The presence of $\mathrm{D}$ band in the graphene spectrum indicates a formation of defect in the graphene structure. We note that the mean intensity ratio of D and G bands $\left(I_{\mathrm{D}} / I_{\mathrm{G}}\right)$ for the graphene is 0.42 and the value actually is not far off from the mean $I_{\mathrm{D}} / I_{\mathrm{G}}$ of graphite $(0.048)$. This observation shows that the defects presence in our exfoliated

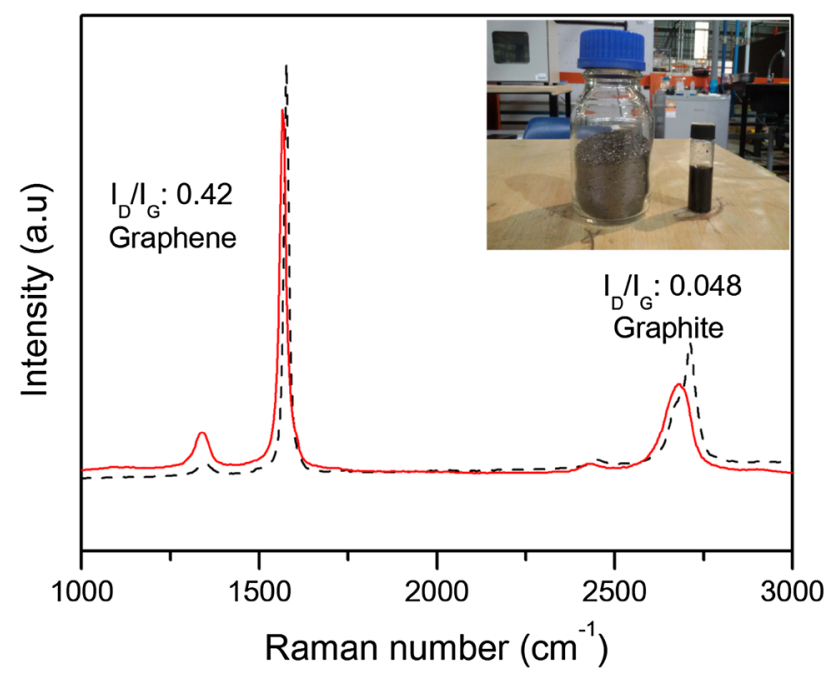

Fig. 3 Raman spectra for graphite and graphene graphene is not similar to the defects level in reduced graphene with $I_{\mathrm{D}} / I_{\mathrm{G}}$ of 1 [25]. Furthermore, the low $I_{\mathrm{D}} /$ $I_{\mathrm{G}}$ for collected graphene can also be used as indicator that exfoliation during mixing action did not induce any significant defects on the graphene flakes as been suggested from previous TEM imaging study. We notice that for graphite the existing defect is not major and did not influence the resulting defects in exfoliated graphene. From the derived equation in the previous work by groups of Coleman [26], we also performed an estimation of mean length for resulting few-layers graphene using the obtained Raman data. Taken the value of FWHM for G-band as $21.66 \mathrm{~cm}^{-1}$, we estimate that the mean length of produced graphene in this work was $529.1 \mathrm{~nm}$ with $20 \%$ of deviation.

\section{Shear functionalization of graphene}

We conducted X-ray photon spectroscopy (XPS) on the vacuum-filtered graphene film for the characterisation of graphene structure after exfoliation. Table 1 shows the composition difference between graphite and exfoliated graphene after the mixing. As shown in Fig. 4a, only oxygen $(532.5 \mathrm{eV})$, silicon $(103.5 \mathrm{eV})$ and carbon $(284.5 \mathrm{eV})$ are present in the survey spectrum of initial graphite sample. After exfoliation, however, additional change, such as low nitrogen presence $(400.5 \mathrm{eV})$ and increase of oxygen intensity, is observed for XPS analysed $G_{\mathrm{GA}}$ (see Fig. 4b). These chemical transformations of exfoliated graphene were possibly caused by the adsorption of gum Arabic on the graphene sheets [22]. Based on the calculated carbon to oxygen $(\mathrm{C} / \mathrm{O})$ ratio, we note that $\mathrm{C} / \mathrm{O}$ value of $G_{\mathrm{GA}}$ is above the reported $\mathrm{C} / \mathrm{O}$ for graphene oxide (2.1-2.6) but is actually far from the published $\mathrm{C} / \mathrm{O}$ for reduced graphene oxide (7.1-10.3) [27]. High oxygen content in $G_{\mathrm{GA}}$ is indicating that large quantity of gum Arabic is required for stabilisation of graphene sheets against reaggregation to graphite in a liquid. To address this assumption, we investigated the chemical change of graphene from the presented carbon $1 \mathrm{~s}(\mathrm{C} 1 \mathrm{~s})$ core of graphite and $\mathrm{C} 1 \mathrm{~s}$ core of graphene in Fig. $4 \mathrm{c}, \mathrm{d}$. In the original graphite, $\mathrm{C}=\mathrm{C} \mathrm{sp}{ }^{2}(284.5 \mathrm{eV})$ and $\mathrm{C}-\mathrm{C} \mathrm{sp}{ }^{3}(285.3 \mathrm{eV})$ are

Table 1 Concentrations of atoms for $G_{\mathrm{GA}}$ and graphite from XPS study

\begin{tabular}{llllll}
\hline \multicolumn{7}{l}{ Atomic composition in (\%) } & & \multicolumn{2}{c}{ C/O ratio } \\
\cline { 1 - 3 } Sample & $\mathrm{C}$ & $\mathrm{O}$ & $\mathrm{Si} 2 \mathrm{p}$ & $\mathrm{N}$ & \\
\hline$G_{\mathrm{GA}}$ & 69.97 & 23.63 & 4.23 & 2.17 & $2.96-3$ \\
Graphite & 92.37 & 5.87 & 1.75 & - & $15.74-16$ \\
\hline
\end{tabular}

The $\mathrm{C} / \mathrm{O}$ ratio was used to show the change of molecular structure for graphene after exfoliation by mixer 
Fig. 4 a and $\mathbf{b}$ show the collected survey spectra from graphite and graphene while c and $\mathbf{d}$ are corresponding to the $\mathrm{C} 1 \mathrm{~s}$ core of graphite and graphene. e FTIR spectra of graphite, gum Arabic and graphene
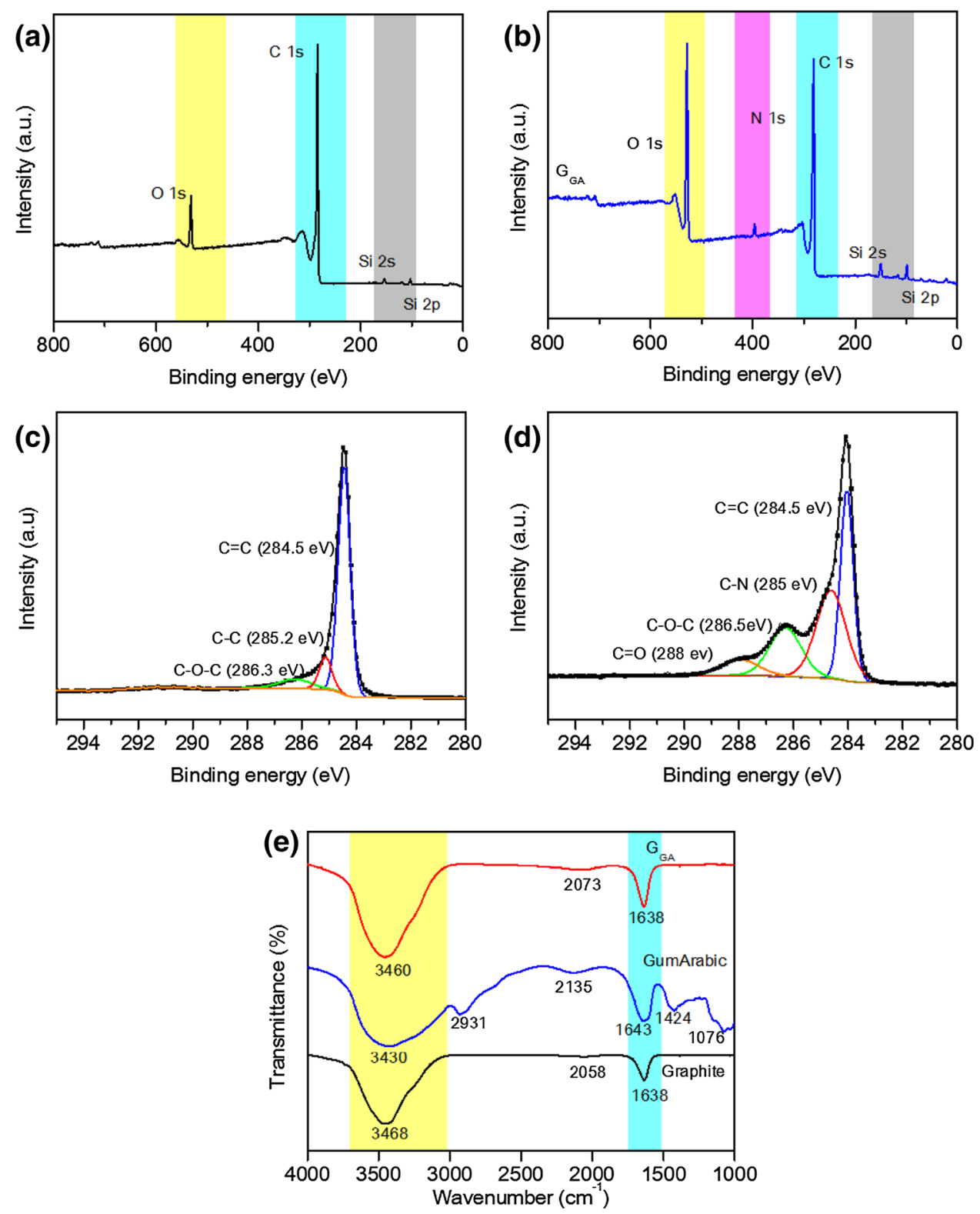

the only major groups that form the chemical structure of graphite. After exfoliation, the formations of, $\mathrm{C}-\mathrm{N}, \mathrm{C}-\mathrm{O}-\mathrm{C}$ and $\mathrm{C}=\mathrm{O}$ groups besides the original $\mathrm{C}=\mathrm{C}$ and $\mathrm{C}-\mathrm{C}$ in the composition of exfoliated graphene are matched with the additional presence of three peaks at, 285, 286.5 and $288 \mathrm{eV}$ [22]. This particular observation confirms that oxygen groups are majorly responsible for stability of graphene against reaggregation in gum Arabic solution.

Figure $4 \mathrm{e}$ shows the measured spectra of graphite, gum Arabic and $G_{\mathrm{GA}}$ from Infrared Spectroscopy (IR). The stretching vibrations at $1638 \mathrm{~cm}^{-1}$ correspond to $\mathrm{C}=\mathrm{O}$ bonds in graphite and graphene. The broadening peak at $3460 \mathrm{~cm}^{-1}$ for $G_{\mathrm{GA}}$ and $3468 \mathrm{~cm}^{-1}$ for graphite meanwhile is originating from the presence of hydrogen bonded $\mathrm{OH}$ groups in both samples. The absorption band at
$2931 \mathrm{~cm}^{-1}$ in IR spectrum of gum Arabic suggests the strong presence of sugar galactose, arabinose and rhamnose (Arabinogalactan) in the material [28]. Weak symmetric stretching at the band $1424 \mathrm{~cm}^{-1}$ is mainly due to the $\mathrm{C}=\mathrm{O}$ of glucuronic acid while the absorption band at $1076 \mathrm{~cm}^{-1}$ highlights the plausible presence of alkene $\mathrm{C}-\mathrm{H}$ bonds for existing polysaccharides in gum Arabic. We note that the disappearance of IR band at $1076 \mathrm{~cm}^{-1}$ in graphene spectrum may be due to the breaking of polysaccharide bonds during shear functionalization of gum Arabic with graphene. The loss of FTIR peak at 1424 and $2931 \mathrm{~cm}^{-1}$ for graphene meanwhile is attributed to the adsorption mechanism of gum Arabic on graphene that is involving carbohydrate and sugar groups. Stronger peaks for graphene at 3460 and $1638 \mathrm{~cm}^{-1}$ though indicate the increase 
of oxygen content in graphene structure after exfoliation by a kitchen mixer.

\section{Yield and stability of exfoliated graphene in water}

To study the yield concentration of produced graphene by gum Arabic-assisted exfoliation, we varied the mass of gum Arabic (1-5 g) and graphite (2.5 and $5.0 \mathrm{~g}$ ) in $400 \mathrm{ml}$ of volume. The kitchen mixer was operated at $15 \mathrm{~min}$ for each individual set of experiment. As shown in Fig. 5a, the mass of initial graphite is important to secure higher concentration yield of graphene. While the maximum yield of graphene for $2.5 \mathrm{~g}$ of graphite was only achieved at $3.0 \mathrm{~g}$ of gum Arabic, the increase of initial graphite to $5.0 \mathrm{~g}$ of graphite would improve the yield concentration even after incorporation $5.0 \mathrm{~g}$ of gum Arabic. For further investigation of this phenomenon, we replotted the data into a new graph with the concentration ratio of graphite to gum Arabic $\left(C_{\mathrm{iG}} / C_{\mathrm{GA}}\right)$ taken as $x$-axis. It was found from Fig. $5 \mathrm{~b}$ that the optimum production of graphene was only possible at the specific value of ratio $\left(C_{\mathrm{iG}} / C_{\mathrm{GA}}=1\right)$. As reported previously [11], this ratio is influential for efficient production of graphene by a kitchen mixer and must be considered prior of any exfoliation stage. In our case, the increase of graphite mass must be always accompanied by the similar mass increase of gum Arabic. The yield efficiency $(\%)$ for our method meanwhile was computed from the highest yield concentration data obtained in this study
( $\sim 17 \mu \mathrm{g} / \mathrm{ml})$. We found that our yield value $(0.136 \%)$ is comparable with the reported yield of graphene $(0.1 \%)$ from shear exfoliation by homogenizer [10] and a kitchen mixer [11].

To evaluate the stability of produced graphene in water, we have to perform triple measurements of absorbance for each supernatant and dispersion of freeze-dried graphene at every $24 \mathrm{~h}$ for 6 days (see Fig. 5c). From the unchanged values of absorbance for supernatant throughout the measurement, it is suggested that the resulting graphene after exfoliation and centrifugation stage is very stable in water. However, we found that the redispersion of graphene into water would result in rapid sedimentation of sheets as been indicated from the continuous drop of absorbance within 6 days. We note, however, that our finding is consistent with the published result on the sedimentation study of polysaccharide-graphene after redispersion in water [29].

\section{Mechanism in exfoliation of graphene by a kitchen mixer}

\section{Reduction of surface tension by gum Arabic}

During the exfoliation of graphite to graphene, the presence of gum Arabic is required to promote the reduction of water surface tension. This is critical as it allows the surface tension of water to match the surface tension of graphene. By adjusting the surface tension value between gum
Fig. 5 a Effect of gum Arabic mass on the yield concentration of graphene b Determination of optimum ratio between graphite and gum Arabic mass c Stability of graphene in water after redispersion as compared to that of decanted supernatant
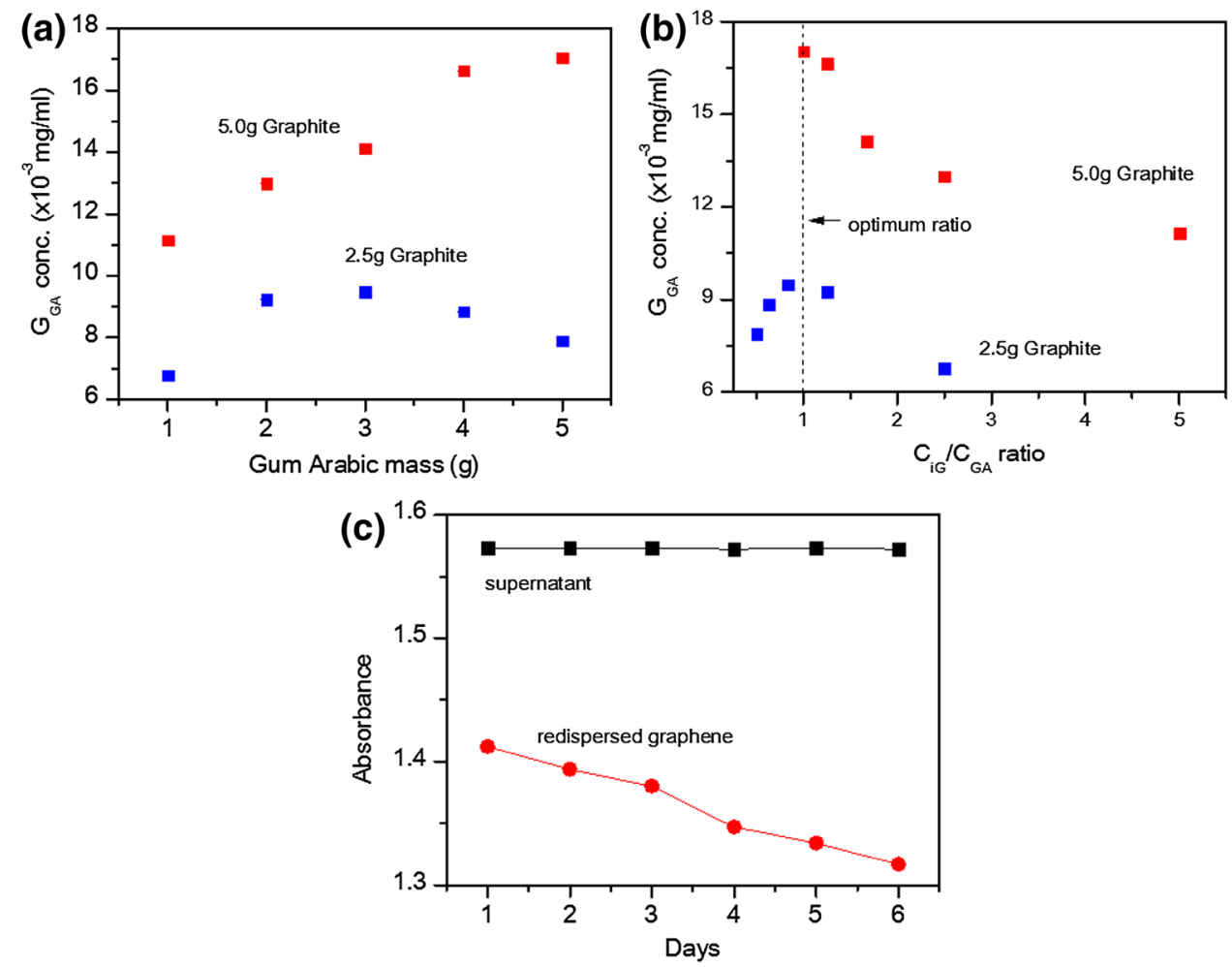
Arabic/water solution and graphite surface, the wetting capability of graphite is gradually increased from the adsorption of gum Arabic on graphene sheets [22]. As suggested by the data of graphene from XPS and IR, the adsorptions of chemical components from gum Arabic are mainly responsible for shear exfoliation and stabilisation of graphene in water. To confirm that the wetting of graphite is still the main mechanism for graphene exfoliation in a kitchen mixer, we have conducted contact angle measurements for both graphite and graphene. As shown in Fig. 6a, the contact angle of graphite $\left(90^{\circ}\right)$ is larger than that of graphene $\left(75^{\circ}\right)$ and is consistent with the previous reported work on sonication of graphene [22]. This is expected as it is known that graphite is originally hydrophobic in nature and the wetting mechanism is independent of selected preparation method. As suggested by previous work [30], the presence of surfactant in the chemical structure of
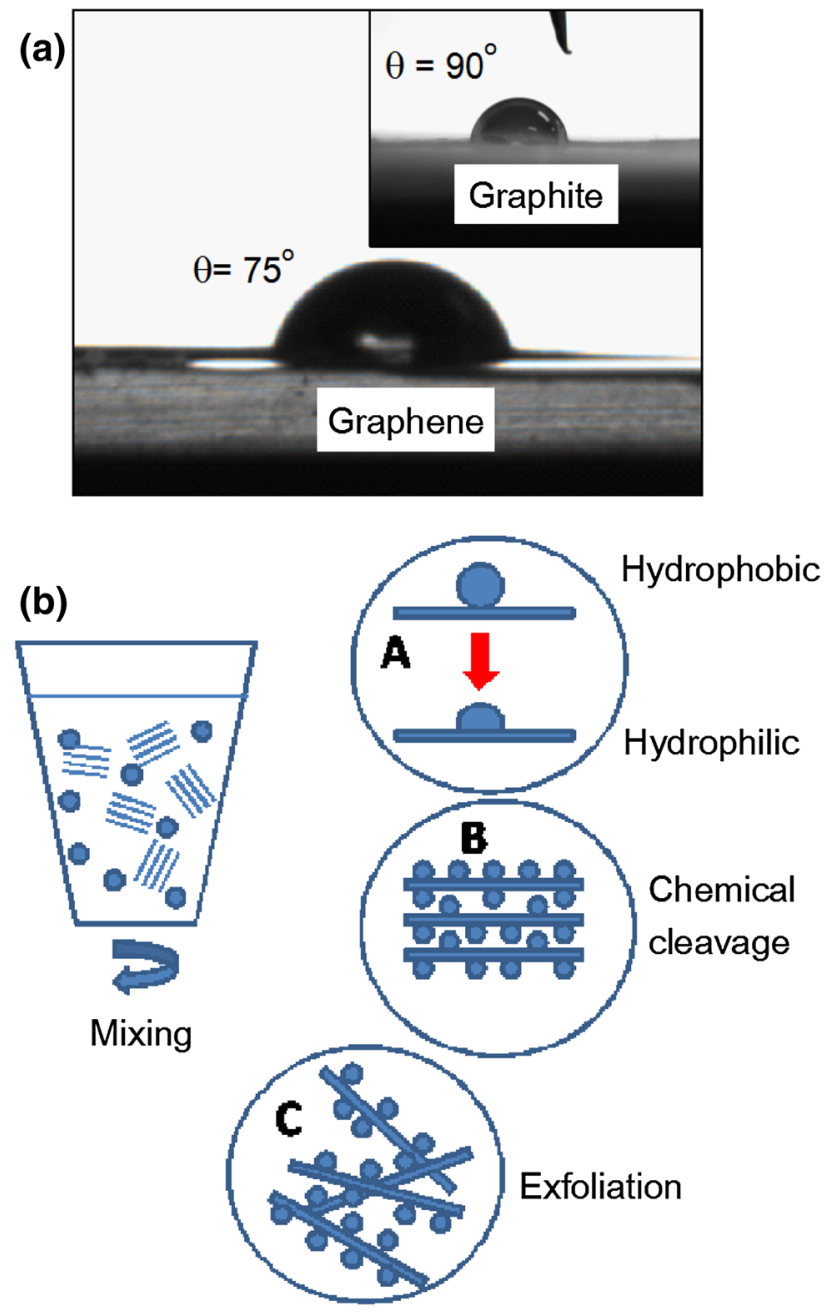

Fig. 6 a Contact angle measurement of water droplet on graphite and graphene film surface. The change of contact angle for graphene indicates the increase of wetting behaviour after adsorption of gum Arabic, b we describe exfoliation mechanism of graphite in gum Arabic in three stages graphene through $\pi$ to $\pi$ mechanism can reduce the hydrophobicity of graphite and resulting in the exfoliation to graphene (see Fig. 6b).

From the 2nd scenario model proposed in the Paton's work [10], the reduction of water surface tension during exfoliation event can be investigated by the following expression:

$\dot{\gamma}_{\min }=\frac{\left(\sqrt{E_{\mathrm{S}, \mathrm{G}}}-\sqrt{E_{\mathrm{S}, \mathrm{L}}}\right)^{2}}{\eta L}$,

where $\dot{\gamma}_{\min }$ is the minimum local shear rate required for exfoliation of graphene, $\sqrt{E_{\mathrm{S}, \mathrm{G}}}$ and $\sqrt{E_{\mathrm{S}, \mathrm{L}}}$ are the graphene and water surface energy, respectively, $\eta$ is the dynamic viscosity of water whilst $L$ is the corresponding length of exfoliated graphene at minimum shear rate. We note that the pre-determined value of maximum local shear rate in our exfoliation of graphene is $2.7 \times 10^{4} \mathrm{~s}^{-1}$, which was calculated by practically considering the $60 \%$ loss of motor power to surrounding [31]. This maximum shear rate surprisingly is relatively similar to the proposed value of $\dot{\gamma}_{\text {min }}$ [10]. Therefore, we assume that it is safe to replace the value of $L$ with the estimated $L$ of $585.1 \mathrm{~nm}$ from our previous exfoliation stage. By taking the value of $E_{\mathrm{S}, \mathrm{G}}$ as $71 \mathrm{~mJ} / \mathrm{m}^{2}$ [10], $\eta$ as $8.9 \times 10^{-4} \mathrm{~Pa} \mathrm{~s}$ and $\dot{\gamma}_{\min }$ as $1 \times 10^{4} \mathrm{~s}^{-1}$, the actual surface energy of the water during exfoliation $\left(E_{\mathrm{S}, \mathrm{L}}\right)$ was computed as $E_{\mathrm{S}, \mathrm{L}}=70.99783 \mathrm{~mJ} /$ $\mathrm{m}^{2}$.

The surface tension of liquid, $\Gamma$, therefore can be expressed in terms of surface energy as [32]:

$\Gamma=E_{\mathrm{S}, \mathrm{L}}-T \cdot S_{\mathrm{S}, \mathrm{L}}$

where $T$ is the temperature in Kelvin, whilst $S_{\mathrm{S}, \mathrm{L}}$ is the surface entropy for the liquid. As surface entropy of water is $0.07 \mathrm{~mJ} / \mathrm{m}^{2} \mathrm{~K}$, the required surface tension of water at room temperature is known as $\Gamma=49.9978 \mathrm{~mJ} / \mathrm{m}^{2}$. We note that this obtained value is actually below the surface tension of pure water at ambient $\left(72.8 \mathrm{~mJ} / \mathrm{m}^{2}\right)$ and is in range with the reported surface tension of NMP [10] and gum Arabic at $3 \mathrm{wt} \%$ of concentrations $\left(55 \mathrm{~mJ} / \mathrm{m}^{2}\right)$ [33]. Based on this observation, we suggest that the surface tension of water must be within this value prior the sliding of graphite layer. We found, however, that due to the dynamic change of water surface tension after surfactant addition, Eq. (2) is only applicable for determination of surface tension for solvent [34].

In principle, the adsorption of gum Arabic on graphene can be described by $\pi-\pi$ stacking mechanism of surfactant on the interlayer graphite [30]. We suggest that the protein components of gum Arabic in arabinogalactan-protein (AGP) and glycoprotein (GP) are chemically attached on the graphite surface as the efficiency of solid-liquid interfaces is increased due to the amphiphilic nature of 
AGP and GP components. Polar component of AGP and GP (polysaccharide) meanwhile attach themselves with water molecules in the medium [35]. Colloidal suspension of graphene provides the stability of gum Arabic-graphene after adsorption of gum Arabic on the surface. The assumption of protein as the responsible molecules for exfoliation of graphene is validated from the presented data in previous XPS and IR results. The addition of $\mathrm{C}=\mathrm{O}$ and $\mathrm{C}-\mathrm{N}$ in the sample after exfoliation stage indicates the adsorption of protein on the graphene surface.

\section{Exfoliation model of graphite}

Using Paton's model, which actually was developed for exfoliation mechanism of graphene in a solvent, we assume that the exfoliation of graphene is initiated after the adsorption of surfactant on the graphite sheets by wetting mechanism. This is of course plausible as we know that the exfoliation of graphene in surfactant medium is triggered after the adsorption of gum Arabic [22] or pyrenecarboxylic acid [30] on the graphite surface. With water as exfoliating medium, the adsorption of gum Arabic on graphite surface is only possible once the surface tension of water is reduced to match to that of graphite. It is predicted that the graphite hydrophobicity will gradually decrease towards hydrophilicity after the increase of gum Arabic presence on the graphite sheets. This increased hydrophilicity will further improve the wetting behaviour of graphite in water. The interfacial bonding energy between surfactant layer on the graphite and liquid becomes stronger than the interfacial bonding between graphite sheets by van der Waals forces. The exfoliation of graphite finally is initiated through sliding mechanism of top layer graphite from the driving forces by shear energy of a kitchen mixer.

In this work, however, we would like to discuss the role of rotational motion in the exfoliation mechanism of graphite by a kitchen mixer [12]. We note that the rotational motion aside translational may be applied on the graphite sheets during the pumping cycle of liquids in the mixing vessel. By considering the presence of $\tau$ and $T$, the mechanism schematic for exfoliation of graphite is graphically presented in Fig. 7.

To develop this simple model, we assume the geometry of graphite as a composition of multiple circular discs and each single disk is fixed at one end by the van der Waals (vdw) forces from the adjacent disc. Considering that the resulting shear flow of the fluid (moving parallel to each other but at different speed) is applied on the free end of graphite surface, the disk composite will be subjected to torsional forces due to the resultant rotational forces ( $T$ and $T$ ) on each end surfaces (scenario a). We note that the cross-sectional plane of the discs will remain undistorted due to geometry of the composition [36]. However, as the top graphite crystal is only linked by weak vdw forces on one end, the resulting shearing stress $\left(\tau_{\max }\right)$ on the graphite vertical will be used to slip the graphite further until it completes delamination stage (scenario $b$ and c). Since $T=F L / 2$ and $F_{\min }$ can be linked to the minimum local shear rate $\left(\gamma_{\min }\right)$ of Newtonian fluid by [10]:

$F_{\min }=\eta \dot{\gamma}_{\min } L^{2}$

the minimum required forces to initiate the exfoliation stage of graphene from the known values of $\gamma_{\min }, L$ and $\eta$ can be practically computed. In our case, the resultant $T_{\min }$ is $6.586 \times 10^{-19} \mathrm{Nm}$, which is extremely small value for rotational forces required on the graphite. The magnitude of shearing stress $(\tau)$ for exfoliation of individual graphite crystal then is calculated from the given $T_{\min }$ by the following equation [36]:

$\tau=\frac{T L}{2 J}$,

where $J=0.5 \pi(L / 2)^{4}$ is the polar moment of inertia for a solid disk and is established as $7.68 \times 10^{-27} \mathrm{~m}$. The applied shearing stress for slipping of graphite from its original plane is, therefore, known as $22.6 \mathrm{~Pa}$. This value of $\tau$ is certainly not adequate to overcome the computed interlayer strength of graphite in dry state which is evaluated at $0.14 \mathrm{GPa}$ [37]. This is where the critical role of wetting on graphite sheets is highlighted as the interlayer strength of graphite will be reduced greatly due to the low friction level of graphite in wet state [38]. Assuming that our graphite system remains elastic, we can determine the angle of twist produced during the slippage (scenario a) through the application of presented equation [36]:

$\theta=\frac{T t}{J G}$

where $G$ is known as the reported modulus of rigidity for graphene (280 GPa) [39] and $t$ is the thickness of single graphite crystal $(0.335 \mathrm{~nm})$ [2]. The resultant $\theta$ is $1.026 \times 10^{-10}$ radians and this value is indicating that the plane of graphite crystal will rotate about $5.88^{\circ}$ from its original axis in nano-domain to initiate a delamination stage. It is apparent that our proposed model could be used to describe the role of rotational motion during graphite delamination by a kitchen mixer. In spite of this possibility, we admit that the application of disc geometry to represent a single layer of graphite crystal in our proposed model might not be highly accurate. Moreover, we choose to neglect the possible rupture effect of shear flow on graphite crystal, which ultimately may be responsible for fragmentation mechanism of the sheets during exfoliation. 


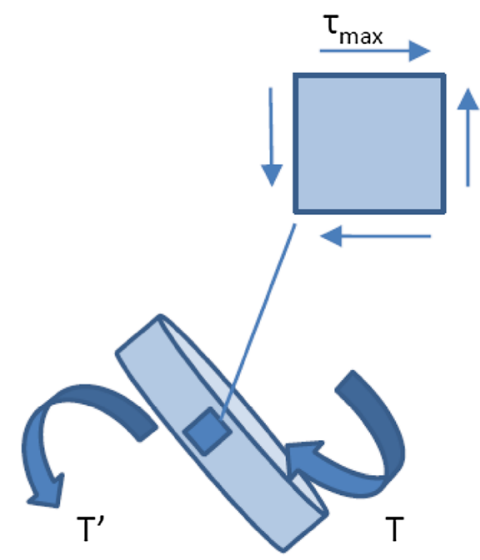

(a)

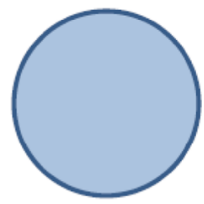

(b)

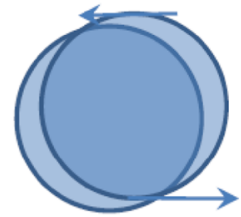

(c)

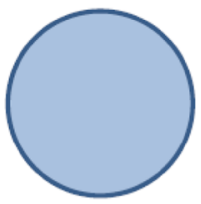

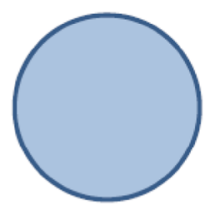

Fig. 7 Exfoliation schematic shows the role of rotation $(T)$ and shear $(\tau)$ for exfoliation of graphite crystal

\section{In situ measurement of graphene size by UV spectroscopy}

Previous works on the sonication [40] and shear mixing of graphene $[12,13]$ have shown the effect of mixing on the size reduction of sheet. Even multiple empirical functions were developed in one work to demonstrate the possibility of UV and Raman for prediction of sheet size and thickness [26]. In their extensive work, we note, however, that the application of UV spectroscopy was used only to estimate the layer number but not the lateral size of graphene. Thus, we would like to propose a simple metric system for evaluation of graphite sheet size from measurement by UV spectroscopy in this paper.

To establish our metric, we note that the sheets size of graphene in our work is strongly dependent on the exfoliation time as shown in Fig. 8a-c. The fragmentation effect of shearing forces on graphene sheets is shown from the increase of graphene number in 300-400 nm range after exfoliation time of $7 \mathrm{~h}$. In our work, we choose not to use centrifugation speed $(\omega)$ for size of selection of our graphene as $\langle\mathrm{L}>$ and $(N)$ of graphene are affected by $\omega$ value [41]. Instead we used variation of exfoliation time and constant $\omega$ to initiate the size change of graphene as only $<\mathrm{L}>$ is strongly dependent on time and not $<\mathrm{N}>$ of graphene sheets [12].

From the resulting UV spectra, the gradual increase of intensity for $\pi-\pi^{*}$ peak (268 $\mathrm{nm}$ ) is indicating the strong of graphene presence after time variation in the supernatant.
We notice that there is no shift of $\pi-\pi^{*}$ peaks, which could be linked to the constant $<\mathrm{N}>$ of graphene sheets in the supernatant [26]. Normalisation of UV spectrum at $\pi-\pi^{*}$ peak, however, reveals the difference in absorbance value from the shoulder (min) and peak point $(\max )$ location is parallel with the size increase of graphene sheets. We estimate that the difference between $\lambda_{\text {peak }}$ and $\lambda_{\text {shoulder }}$ is $(-30 \mathrm{~nm})$ for easier determination of maximum and minimum absorbance of graphene. With this knowledge, the $<\mathrm{L}>$ data were plotted as a function of the absorbance ratio between minimum and maximum of $\pi-\pi^{*}$ peak $\left(\mathrm{Abs}_{\min } / \mathrm{Abs}_{\max }\right)$. Fitting of the data resulting in the metric of $<\mathrm{L}>$ is:

$<\mathrm{L}>=1030.1\left(\frac{\mathrm{Abs}_{\min }}{\mathrm{Abs}_{\max }}\right)^{1.36}$.

While this developed metric may be useful for estimation of sheet size after exfoliation stage, we note however that the value of $\mathrm{Abs}_{\min } / \mathrm{Abs}_{\max }$ must not exceed 0.76. We evaluate the practicality of the proposed metric from the comparison of $<\mathrm{L}>$ after $1 \mathrm{~h}$ exfoliation time, resulting in $<\mathrm{L}>$ of $761 \mathrm{~nm}$ from the metric and $<\mathrm{L}>$ of $943 \mathrm{~nm}$ from the imaging by TEM for $\mathrm{Abs}_{\min } / \mathrm{Abs}_{\max }=0.8$. This limitation is possibly caused by the exponential change of graphene size with exfoliation duration that is lower than $180 \min$ [12].

Based on the produced TEM data, the minimum size of our graphene is measured at $200 \mathrm{~nm}$ and we know that the value of $\mathrm{Abs}_{\min } / \mathrm{Abs}_{\max }$ is beyond the scope of our metric. 
Fig. 8 a, b and $\mathbf{c}$ show the change of graphene size after the increase of exfoliation time, (d) The increase of $\pi-\pi^{*}$ peak is indicating the improvement of graphene concentration in collected supernatant, (e) After normalisation at local maximum, we note a shift of absorbance value at UV shoulder, (f) Empirical function of $<\mathrm{L}>$ as the value of $\mathrm{Abs}_{\min }$ l $\mathrm{Abs}_{\max }$
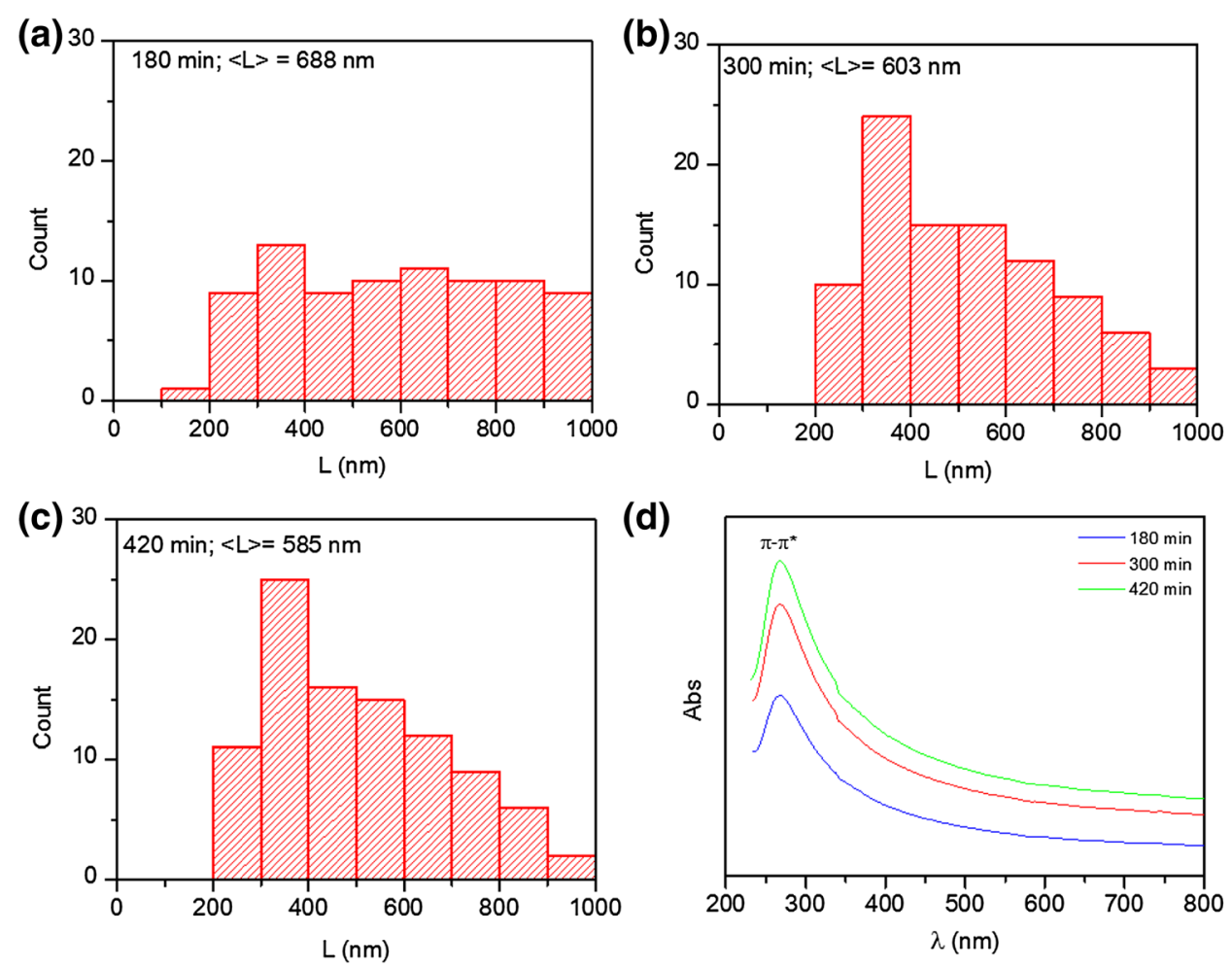

(d)

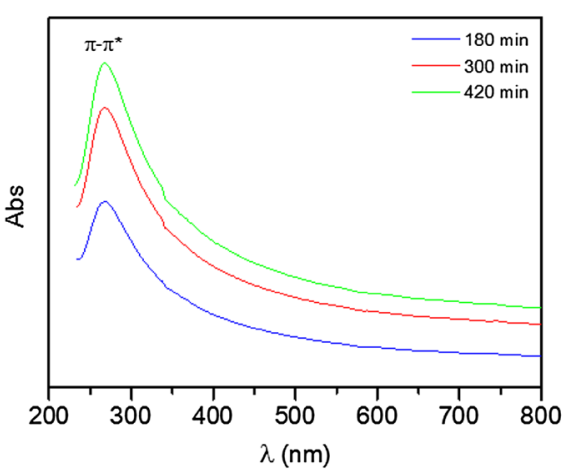

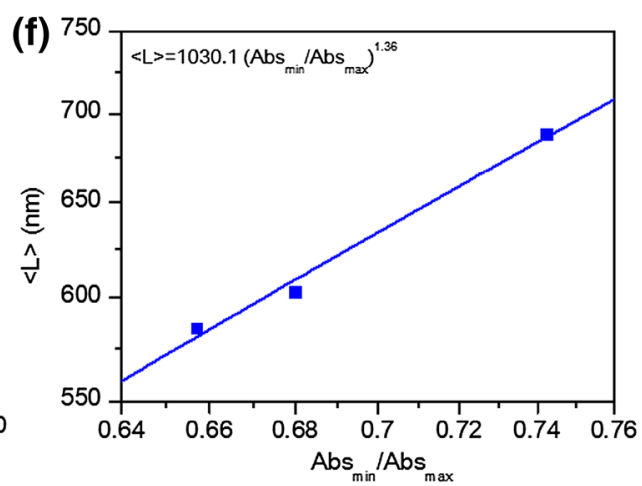

However, we would like to propose a modification of our metric, which may be suitable for prediction of sheet size smaller than $561 \mathrm{~nm}\left(\mathrm{Abs}_{\min } / \mathrm{Abs}_{\max }=0.64\right)$ as:

$<\mathrm{L}>=561\left[1-\left(0.64-\frac{\mathrm{Abs}_{\min }}{\mathrm{Abs}_{\max }}\right)^{1.36}\right]$.

From the presented metric, the possible smallest size of graphene is computed as $255 \mathrm{~nm}$, which is not far from the smallest sheet size determined by our TEM imaging. We do believe that the application of both metrics would be useful for in situ measurement of sheet size in future exfoliation stage, as these metrics allow user to evaluate the resulting $<\mathrm{L}>$ of produced graphene using only UV spectroscopy. In spite of this advantage, we would recommend for further length investigation by TEM after each prediction for validation and confirmation of proposed $<\mathrm{L}>$ value.

\section{Conclusion}

As conclusion, we have shown that it is possible to produce gum Arabic-modified graphene from the co-mixing of graphite and gum Arabic in a commercial kitchen mixer. Through the application of XPS and IR, we have identified the role of protein and polysaccharide role in the stabilisation of graphene in water. It is suggested that the amphiphilic protein from AGP and GP groups of gum Arabic is required for the bridging between polar polysaccharide and hydrophobic graphene in water. Through the reduction of surface tension, wetting of graphite is initiated for exfoliation and stabilisation of graphene against reaggregation. In addition, we have proposed an exfoliation model that was designed by considering the rotational motion of graphite during exfoliation to graphene. The generated model used to describe the 
exfoliation of graphene sheet from graphite with the magnitude of $\tau$ and $\theta$ was computed from the application of basic continuum mechanics. To accommodate these simple calculations, we must assume the geometry of our bulky graphite as an attached circular disk. For in situ evaluation of graphene sheet size after exfoliation stage, two simple metrics were developed from the combined application of UV spectroscopy and TEM imaging. However, we note that although we could estimate the sheet size from the application of both metrics, we could not be absolutely certain that the computed $<\mathrm{L}>$ from the metric is the actual one. Thus, we recommended the application of TEM and AFM for validation of the result after the application of these proposed metrics. In this way, while our metrics may be useful as rapid measurement tool for $\langle\mathrm{L}>$ of graphene after exfoliation, the resulting data from TEM or AFM could still be used as supporting data for later validation step.

Acknowledgements This research has received funding from Ministry of Higher Education of Malaysia and Universiti Malaysia Pahang under grant agreement of RDU 160149 and ULC 150306. In addition, we are grateful for assistance provided by Universiti Sains Malaysia (SERC) and Universiti Putra Malaysia (ITMA) during XPS and Raman characterisation. We are also very thankful to Mr. Fahmi from Universiti Malaysia Pahang (CARIFF) for technical support during the imaging of graphene by TEM.

Open Access This article is distributed under the terms of the Creative Commons Attribution 4.0 International License (http://crea tivecommons.org/licenses/by/4.0/), which permits unrestricted use, distribution, and reproduction in any medium, provided you give appropriate credit to the original author(s) and the source, provide a link to the Creative Commons license, and indicate if changes were made.

\section{References}

1. Geim, A.K., Novoselov, K.S.: The rise of graphene. Nat. Mater. 6(3), 183-191 (2007)

2. Lee, C., Wei, X., Kysar, J.W., Hone, J.: Measurement of the elastic properties and intrinsic strength of monolayer graphene. Science 321(5887), 385-388 (2008)

3. Abidin, A.S.Z., Yusoh, K., Jamari, S.S., Abdullah, A.H., Ismail, Z.: Enhanced performance of alkylated graphene reinforced polybutylene succinate nanocomposite. AIP Conf. Proc. 1756(1), 040005 (2016). doi:10.1063/1.4958766

4. Kang, M.S., Kim, K.T., Lee, J.U., Jo, W.H.: Direct exfoliation of graphite using a non-ionic polymer surfactant for fabrication of transparent and conductive graphene films. J. Mater. Chem. C 1(9), 1870-1875 (2013)

5. Surwade, S.P., Smirnov, S.N., Vlassiouk, I.V., Unocic, R.R., Veith, G.M., Dai, S., Mahurin, S.M.: Water desalination using nanoporous single-layer graphene. Nat. Nanotechnol. 10(5), 459-464 (2015)

6. Huang, L., Huang, Y., Liang, J., Wan, X., Chen, Y.: Graphenebased conducting inks for direct inkjet printing of flexible conductive patterns and their applications in electric circuits and chemical sensors. Nano Res. 4(7), 675-684 (2011)
7. Andryieuski, A., Lavrinenko, A.V., Chigrin, D.N.: Graphene hyperlens for terahertz radiation. Phys. Rev. B 86(12), 121108 (2012)

8. Yan, K., Fu, L., Peng, H., Liu, Z.: Designed CVD growth of graphene via process engineering. Acc. Chem. Res. 46(10), 2263-2274 (2013)

9. Sutter, P.W., Flege, J.-I., Sutter, E.A.: Epitaxial graphene on ruthenium. Nat. Mater. 7(5), 406-411 (2008)

10. Paton, K.R., Varrla, E., Backes, C., Smith, R.J., Khan, U., O'Neill, A., Boland, C., Lotya, M., Istrate, O.M., King, P., Higgins, T., Barwich, S., May, P., Puczkarski, P., Ahmed, I., Moebius, M., Pettersson, H., Long, E., Coelho, J., O’Brien, S.E., McGuire, E.K., Sanchez, B.M., Duesberg, G.S., McEvoy, N., Pennycook, T.J., Downing, C., Crossley, A., Nicolosi, V., Coleman, J.N.: Scalable production of large quantities of defect-free few-layer graphene by shear exfoliation in liquids. Nat. Mater. 13(6), 624-630 (2014). doi:10.1038/nmat3944

11. Varrla, E., Paton, K.R., Backes, C., Harvey, A., Smith, R.J., McCauley, J., Coleman, J.N.: Turbulence-assisted shear exfoliation of graphene using household detergent and a kitchen blender. Nanoscale 6(20), 11810-11819 (2014)

12. Yi, M., Shen, Z.: Kitchen blender for producing high-quality fewlayer graphene. Carbon 78, 622-626 (2014)

13. Pattammattel, A., Kumar, C.V.: Kitchen chemistry 101: multigram production of high quality biographene in a blender with edible proteins. Adv. Funct. Mater. 25(45), 7088-7098 (2015)

14. Liu, L., Shen, Z., Yi, M., Zhang, X., Ma, S.: A green, rapid and size-controlled production of high-quality graphene sheets by hydrodynamic forces. RSC Adv. 4(69), 36464-36470 (2014)

15. Chen, X., Dobson, J.F., Raston, C.L.: Vortex fluidic exfoliation of graphite and boron nitride. Chem. Commun. 48(31), 3703-3705 (2012)

16. Wahid, M.H., Eroglu, E., Chen, X., Smith, S.M., Raston, C.L.: Functional multi-layer graphene-algae hybrid material formed using vortex fluidics. Green Chem. 15(3), 650-655 (2013)

17. Shen, Z., Li, J., Yi, M., Zhang, X., Ma, S.: Preparation of graphene by jet cavitation. Nanotechnology 22(36), 365306 (2011)

18. Yi, M., Li, J., Shen, Z., Zhang, X., Ma, S.: Morphology and structure of mono-and few-layer graphene produced by jet cavitation. Appl. Phys. Lett. 99(12), 123112 (2011)

19. Yi, M., Shen, Z., Zhang, W., Zhu, J., Liu, L., Liang, S., Zhang, X., Ma, S.: Hydrodynamics-assisted scalable production of boron nitride nanosheets and their application in improving oxygenatom erosion resistance of polymeric composites. Nanoscale 5(21), 10660-10667 (2013)

20. Ismail, Z., Abdullah, A.H., Zainal Abidin, A.S., Yusoh, K.: Application of graphene from exfoliation in kitchen mixer allows mechanical reinforcement of PVA/graphene film. Appl. Nanosci. (2017). doi:10.1007/s13204-017-0574-y

21. Chabot, V., Kim, B., Sloper, B., Tzoganakis, C., Yu, A.: High yield production and purification of few layer graphene by Gum Arabic assisted physical sonication. Sci. Rep. 3, 1378 (2013)

22. Fan, J., Shi, Z., Ge, Y., Wang, J., Wang, Y., Yin, J.: Gum arabic assisted exfoliation and fabrication of Ag-graphene-based hybrids. J. Mater. Chem. 22(27), 13764-13772 (2012)

23. Paton, K.R., Coleman, J.N.: Relating the optical absorption coefficient of nanosheet dispersions to the intrinsic monolayer absorption. Carbon 107, 733-738 (2016). doi:10.1016/j.carbon. 2016.06.043

24. Su, R., Lin, S.F., Chen, D.Q., Chen, G.H.: Study on the absorption coefficient of reduced graphene oxide dispersion. J. Phys. Chem. C 118(23), 12520-12525 (2014). doi:10.1021/jp500499d

25. Gómez-Navarro, C., Weitz, R.T., Bittner, A.M., Scolari, M., Mews, A., Burghard, M., Kern, K.: Electronic transport properties of individual chemically reduced graphene oxide sheets. Nano Lett. 7(11), 3499-3503 (2007). doi:10.1021/n1072090c 
26. Backes, C., Paton, K.R., Hanlon, D., Yuan, S., Katsnelson, M.I., Houston, J., Smith, R.J., McCloskey, D., Donegan, J.F., Coleman, J.N.: Spectroscopic metrics allow in situ measurement of mean size and thickness of liquid-exfoliated few-layer graphene nanosheets. Nanoscale 8(7), 4311-4323 (2016)

27. Stobinski, L., Lesiak, B., Malolepszy, A., Mazurkiewicz, M., Mierzwa, B., Zemek, J., Jiricek, P., Bieloshapka, I.: Graphene oxide and reduced graphene oxide studied by the XRD, TEM and electron spectroscopy methods. J. Electron Spectrosc. Relat. Phenom. 195, 145-154 (2014)

28. Daoub, R.M.A., Elmubarak, A.H., Misran, M., Hassan, E.A., Osman, M.E.: Characterization and functional properties of some natural Acacia gums. J. Saudi Soc. Agric. Sci. (2016). doi:10. 1016/j.jssas.2016.05.002

29. Unalan, I.U., Wan, C., Trabattoni, S., Piergiovanni, L., Farris, S.: Polysaccharide-assisted rapid exfoliation of graphite platelets into high quality water-dispersible graphene sheets. RSC Adv. 5(34), 26482-26490 (2015)

30. An, X., Simmons, T., Shah, R., Wolfe, C., Lewis, K.M., Washington, M., Nayak, S.K., Talapatra, S., Kar, S.: Stable aqueous dispersions of noncovalently functionalized graphene from graphite and their multifunctional high-performance applications. Nano Lett. 10(11), 4295-4301 (2010). doi:10.1021/n1903557p

31. Xie, L., Rielly, C.D., Özcan-Taşkin, G.: Break-up of nanoparticle agglomerates by hydrodynamically limited processes. J. Dispers. Sci. Technol. 29(4), 573-579 (2008)

32. Lyklema, J.: The surface tension of pure liquids. Colloids Surf. A 156(1), 413-421 (1999). doi:10.1016/S0927-7757(99)00100-4

33. Cao, C., Zhang, L., Zhang, X.-X., Du, F.-P.: Effect of gum arabic on the surface tension and surface dilational rheology of trisiloxane surfactant. Food Hydrocoll. 30(1), 456-462 (2013). doi:10.1016/j.foodhyd.2012.07.006

34. Birdi, K.: Handbook of surface and colloid chemistry. CRC Press, Boca Raton (2015)

35. Renard, D., Lepvrier, E., Garnier, C., Roblin, P., Nigen, M., Sanchez, C.: Structure of glycoproteins from Acacia gum: an assembly of ring-like glycoproteins modules. Carbohydr. Polym. 99, 736-747 (2014). doi:10.1016/j.carbpol.2013.08.090

36. Beer, F.P., Johnston, E.R., DeWolf, J.T., Mazurek, D.F.: Mechanics of materials. McGraw-Hill Education, New York (2015)

37. Liu, Z., Zhang, S.-M., Yang, J.-R., Liu, J.Z., Yang, Y.-L., Zheng, Q.-S.: Interlayer shear strength of single crystalline graphite. Acta Mech. Sinica 28(4), 978-982 (2012). doi:10.1007/s10409-0120137-0

38. Lancaster, J.: A review of the influence of environmental humidity and water on friction, lubrication and wear. Tribol. Int. 23(6), 371-389 (1990)

39. Liu, X., Metcalf, T.H., Robinson, J.T., Houston, B.H., Scarpa, F.: Shear modulus of monolayer graphene prepared by chemical vapor deposition. Nano Lett. 12(2), 1013-1017 (2012). doi:10. 1021/n1204196v

40. Ismail, Z., Yusoh, K.: Facile method for liquid-exfoliated graphene size prediction by UV-visible spectroscopy. AIP Conf. Proc. 1756(1), 070002 (2016). doi:10.1063/1.4958778

41. Khan, U., O'Neill, A., Porwal, H., May, P., Nawaz, K., Coleman, J.N.: Size selection of dispersed, exfoliated graphene flakes by controlled centrifugation. Carbon 50(2), 470-475 (2012) 